\title{
Engineering entangled-photon states using two-dimensional PPLN crystals
}

\author{
Hugues Guillet de Chatellus ${ }^{a}$, Giovanni Di Giuseppe ${ }^{a}$, Alexander V. Sergienko ${ }^{a, b}$, \\ Bahaa E. A. Saleh ${ }^{a}$, Malvin C. Teich $^{a, b}$, \\ ${ }^{a}$ Quantum Imaging Laboratory, Department of Electrical \& Computer Engineering, Boston \\ University, 8 Saint Mary's Street, Boston, Massachusetts 02215 \\ ${ }^{b}$ Quantum Imaging Laboratory, Department of Physics, Boston University, 8 Saint Mary's \\ Street, Boston, Massachusetts 02215
}

\begin{abstract}
We demonstrate the possibility of using periodically poled lithium niobate crystals (PPLN) as a direct source of entangled photon pairs. Two configurations are studied. The first enables the generation of polarizationentangled states from a one-dimensional PPLN structure at different frequencies; the second is dedicated to the production of frequency-entangled states from a bi-dimensional PPLN structure. The engineering of both of these PPLN structures are described from a theoretical perspective.
\end{abstract}

Keywords: Entanglement, SPDC (spontaneous parametric downconversion), PPLN (periodically poled lithium niobate), ferroelectrics

\section{INTRODUCTION}

Spontaneous parametric downconversion (SPDC) has been widely used as a source of correlated or entangled photon pairs. The applications of these pairs include experiments in quantum mechanics, optical measurements, spectroscopy, imaging, and quantum information. ${ }^{1}$ In a second-order nonlinear medium, SPDC corresponds to the annihilation of a pump photon of energy $\hbar \omega_{p}$ and the creation of two photons named 'signal' and 'idler' whose respective energies are $\hbar \omega_{s}$ and $\hbar \omega_{i}\left(\omega_{s} \geq \omega_{i}\right)$. The frequency, direction of emission, and polarization of the two twin photons are respectively determined by energy conservation, the phase-matching condition, and the type of interaction determined by the symmetry of the crystal. The nonlinear process can be type I or type II where the twin photons have parallel and perpendicular polarizations, respectively. If, by any means, two or more independent states are made indistinguishable in the measurement apparatus, the two-photon state is a coherent superposition of those independent states and the state is entangled.

Up to now, two configurations have been proposed to generate polarization-entangled states directly from the nonlinear crystal, ${ }^{2,3}$ but both were realized in the degenerate case, producing polarization-entangled photons around $800 \mathrm{~nm}$. These are easily detectable by means of standard silicon avalanche photodiodes (APD). For the implementation of quantum communications systems at the telecommunication wavelength, new crystal sources with high downconversion efficiency are necessary, to compensate for the low efficiency of detectors in this wavelength region. The use of periodically poled crystals is an interesting alternative to birefringent phase-matching ${ }^{4}$ or guided-wave phase-matching, ${ }^{5}$ since quasi-phase matching (QPM) has been proven to be an efficient and flexible technique to extend the range of three-wave mixing processes. ${ }^{6,7}$ In the family of commonly used nonlinear media, lithium niobate $\left(\mathrm{LiNbO}_{3}\right)$ boasts one of the largest nonlinear coefficients. ${ }^{8}$ Many of the advantages of QPM in lithium niobate have been outlined elsewhere ${ }^{9}$ and results show that periodically poled lithium niobate (PPLN) is a natural choice for many experiments. PPLN structures have been largely used in the past ten years, mostly for frequency up-conversion, ${ }^{10}$ OPOs,${ }^{8}$ and recently for generating correlated photon pairs. $^{11}$

We demonstrate here that periodically poled lithium niobate is also a convenient nonlinear crystal for the direct generation of polarization-entangled or frequency-entangled pairs of photons, in the highly non-degenerate case where one of the photons is visible and the other is at the telecommunication wavelength. 
First we investigate the properties of the two-photon state generated in a PPLN structure, then we demonstrate the possibility of engineering the patterning of the poling to produce polarization-entangled states and frequency-entangled states. For both cases, we calculate the experimental parameters of these PPLN structures in the specific situation where the crystal is pumped by a beam at $532 \mathrm{~nm}$, and the entangled photons are centered at 810 and $1550 \mathrm{~nm}$.

\section{SPDC IN A PERIODICALLY POLED NONLINEAR CRYSTAL}

In PPLN, the ferroelectric domains are alternatively directed up and down, parallel to the z-axis of the lithium niobate structure. Thus the second order non-linearity tensor is alternatively $+\chi_{i j k}^{(2)}$ and $-\chi_{i j k}^{(2)}$. The effective nonlinear profile seen by the pump wave during its propagation is therefore a square wave function.

Let us consider the general case of a pump beam travelling in a periodically poled non-linear medium characterized by a bi-dimensional nonlinear profile of the form

$$
\chi_{i j k}^{(2)}(\mathbf{r})=\sum_{\mathbf{q}} \tilde{\chi}_{i j k}^{(2)}(\mathbf{q}) e^{-i \mathbf{q} \cdot \mathbf{r}}
$$

where the summation is over all the spatial wave vectors $\mathbf{q}$ arising in the Fourier spectrum of the spatial profile of the nonlinearity. The fact that the Fourier spectrum is discrete comes from the extension of the nonlinear function to the whole space. The fact that the nonlinear medium is actually finite will appear in the integration over the volume of the crystal.

The vectors $\mathbf{q}$ are the spatial harmonics of the modulation. They can be written, in the general case of an infinite bi-dimensional PPLN, as

$$
\mathbf{q}=p_{1} \mathbf{q}_{\mathbf{1}}+p_{2} \mathbf{q}_{\mathbf{2}}
$$

where $\mathbf{q}_{\mathbf{1}}$ and $\mathbf{q}_{\mathbf{2}}$ are the elementary vectors of the reciprocal lattice, $p_{1}$ and $p_{2}$ are integers.

The quantity $\tilde{\chi}_{i j k}^{(2)}(\mathbf{q})$ is the Fourier transform of the nonlinear profile at the spatial wave vector $\mathbf{q}$. By parametric down-conversion, a non-linear crystal occupying a volume $V$ illuminated by an intense pump field emits a signal and an idler photon that can be described by the two-photon state ${ }^{1}$

$$
\left|\psi^{(2)}\right\rangle \sim \int_{V} d \mathbf{r} \int d t \chi_{p s i}^{(2)}(\mathbf{r}) \hat{E}_{p}^{+}(\mathbf{r}, t) \hat{E}_{s}^{-}(\mathbf{r}, t) \hat{E}_{i}^{-}(\mathbf{r}, t)|0\rangle
$$

where $\hat{E}_{s}^{-}$and $\hat{E}_{i}^{-}$are the field operators of the signal and idler respectively:

$$
\begin{aligned}
& \hat{E}_{s}^{-}(\mathbf{r}, t)=\int d \omega_{s} E_{s} e^{-i \mathbf{k}_{\mathbf{s}} \cdot \mathbf{r}} e^{i \omega_{s} t} a_{s}^{+}\left(\omega_{s}, \mathbf{k}_{\mathbf{s}}, s\right) \\
& \hat{E}_{i}^{-}(\mathbf{r}, t)=\int d \omega_{i} E_{i} e^{-i \mathbf{k}_{\mathbf{i}} \cdot \mathbf{r}} e^{i \omega_{i} t} a_{i}^{+}\left(\omega_{i}, \mathbf{k}_{\mathbf{i}}, i\right) .
\end{aligned}
$$

The pump field is given by the complex amplitude

$$
E_{p}^{+}(\mathbf{r}, t)=\int d \omega_{p} E_{p} e^{i \mathbf{k}_{\mathbf{p}} \cdot \mathbf{r}} e^{-i \omega_{p} t} .
$$

The polarization of the three photons are respectively denoted p, s and i. Simple developments lead to :

$$
\left|\psi^{(2)}\right\rangle \sim \int d \omega_{s} d \omega_{p} E_{p} \delta\left(\omega_{p}-\omega_{s}-\omega_{i}\right) \sum_{\mathbf{q}} \tilde{\chi}_{p s i}^{(2)}(\mathbf{q}) \int_{V} d \mathbf{r} e^{i\left(\mathbf{k}_{\mathbf{p}}-\mathbf{k}_{\mathbf{s}}-\mathbf{k}_{\mathbf{i}}-\mathbf{q}\right) \cdot \mathbf{r}} a_{s}^{+}\left(\omega_{s}, \mathbf{k}_{\mathbf{s}}, s\right) a_{i}^{+}\left(\omega_{i}, \mathbf{k}_{\mathbf{i}}, i\right)|0\rangle .
$$




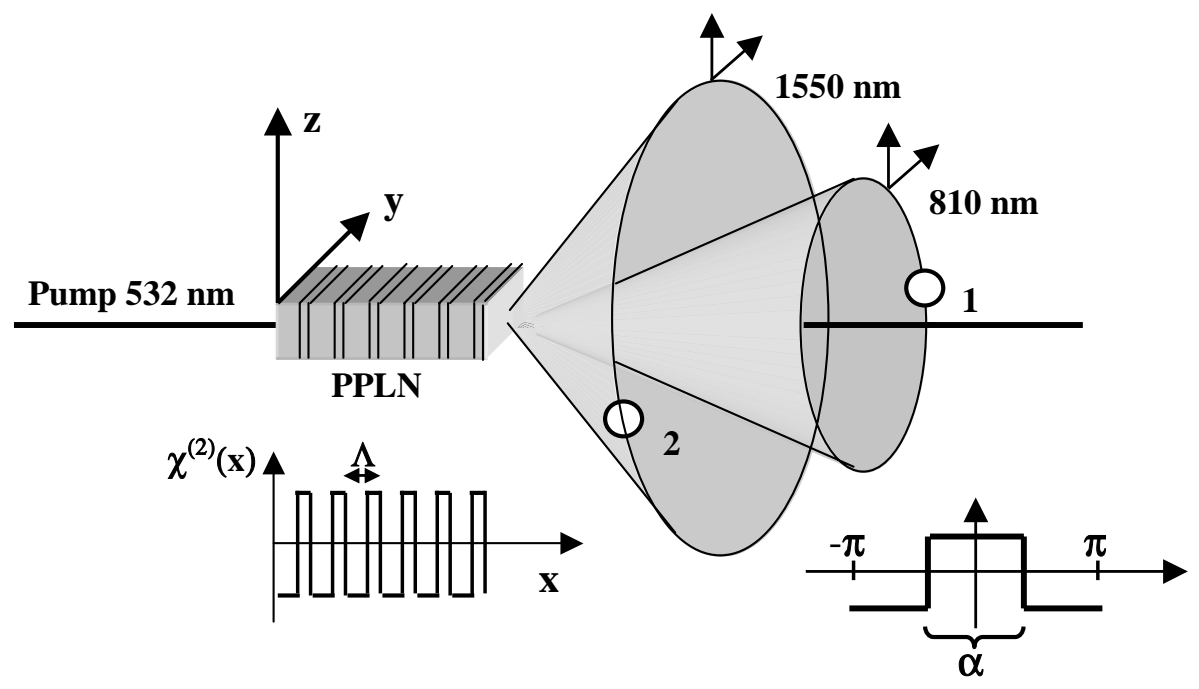

Figure 1. Principle of generating polarization entangled pairs at $810 \mathrm{~nm}$ and $1550 \mathrm{~nm}$. The two systems of cones $(\mathrm{H}$ and $\mathrm{V}$ polarized) overlap. Entangled pairs are collected along the directions labelled 1 and 2 . The two insets show the fundamental parameters of the PPLN: the period $\Lambda$ and the (angular) duty cycle $\alpha$.

The amplitude of the two-photon state does not vanish if both energy conservation and phase-matching condition are satisfied,

$$
\begin{aligned}
& \omega_{p} \sim \omega_{s}+\omega_{i} \\
& \mathbf{k}_{\mathbf{p}}-\mathbf{k}_{\mathbf{s}}-\mathbf{k}_{\mathbf{i}}-\mathbf{q} \sim \mathbf{0} .
\end{aligned}
$$

The latter condition simply means that twin photons satisfying energy conservation can be emitted along $\mathbf{k}_{\mathbf{s}}$ and $\mathbf{k}_{\mathbf{i}}$ if the difference $\mathbf{k}_{\mathbf{p}}-\mathbf{k}_{\mathbf{s}}-\mathbf{k}_{\mathbf{i}}$ matches one of the spatial harmonics of the reciprocal lattice. The amplitude of the corresponding two-photon state is simply proportional to $\tilde{\chi}_{p s i}^{(2)}(\mathbf{q})$. For a square-wave function, harmonics are naturally present in the Fourier spectrum. This leads to the possibility of tailoring the poling of the crystal to engineer (at least) two nonlinear interactions, both satisfying quasi-phase matching conditions where the pairs propagate in coincident directions. In this case, the corresponding states interfere coherently to produce an entangled state. In the following we present the design of PPLN for polarization, and frequency entanglement.

\section{ONE-DIMENSIONAL PPLN FOR POLARIZATION ENTANGLEMENT}

We demonstrate here the practical feasibility of poling a lithium niobate crystal to generate polarization entangled photon pairs at $810 \mathrm{~nm}$ and $1550 \mathrm{~nm}$ from a 532-nm pump beam. This specific interaction was chosen since the $810 \mathrm{~nm}$ photon is readily observed using silicon detectors, and for future realizations in quantum communications at the telecommunication wavelength. A sketch of the interaction and generation is presented in Fig.1.

We consider the following nonlinear interactions :

$$
\begin{aligned}
& 532(e) \longrightarrow 810(e)+1550(e) \\
& 532(e) \longrightarrow 810(o)+1550(o)
\end{aligned}
$$


where $e$ and $o$ stand for extraordinary and ordinary respectively. The respective values of $\chi_{p s i}^{(2)}$ are 32 and $5.8 \mathrm{pm} / \mathrm{V}$.

The intended two-photon entangled state is :

$$
\frac{1}{\sqrt{1+|\zeta|^{2}}}\left(|810, e\rangle_{1}|1550, e\rangle_{2}+\zeta|810, o\rangle_{1}|1550, o\rangle_{2}\right)
$$

where $|\zeta|=1$ for a maximally entangled state. The two main challenges are first to match the directions of emission of the signal and idler coming from the two nonlinear interactions, and second to have the same production rate in the two interactions in order to fulfill $|\zeta|=1$.

\subsection{Matching the directions of emissions of the pairs}

Considering a one-dimensional PPLN structure of period $\Lambda$, and knowing the temperature-dependent Sellmeier equations in lithium niobate for both $e$ and $o$ polarization, we can compute the output angle of the $810 \mathrm{~nm}$ and $1550 \mathrm{~nm}$ photons depending on $|\mathbf{q}|$, the wave vector of the nonlinear profile fulfilling the quasi-phase matching condition in Eq.8. In a $1 \mathrm{D}$ PPLN structure, $\mathbf{q}=p \mathbf{q}_{\mathbf{o}}$ where $q_{o}=2 \pi / \Lambda$.

If the poling pattern possesses a harmonic at $q=0.91 \mathrm{rad} / \mu \mathrm{m}$ and at $q=0$, then the output angles of the 810 and 1550 for the $e \longrightarrow e+e$ interaction that satisfies the quasi-phase matching condition

$$
\mathbf{k}_{\mathbf{5 3 2}}(e)-\mathbf{k}_{\mathbf{8 1 0}}(e)-\mathbf{k}_{\mathbf{1 5 5 0}}(e)=\mathbf{q}=p \mathbf{q}_{\mathbf{0}},
$$

match the output angles for the $e \longrightarrow o+o$ interaction with the phase-matching condition

$$
\mathbf{k}_{\mathbf{5 3 2}}(e)-\mathbf{k}_{\mathbf{8 1 0}}(o)-\mathbf{k}_{\mathbf{1 5 5 0}}(o)=\mathbf{0} .
$$

To produce a $q$ vector equal to $0.91 \mathrm{rad} / \mu \mathrm{m}$, the PPLN can be poled at the fundamental spatial period $(6.9 \mu \mathrm{m})$, or at any larger harmonic $\left(2^{\text {nd }}\right.$ at $13.7 \mu \mathrm{m}, 3^{r d}$ at $20.6 \mu \mathrm{m}, 4^{\text {th }}$ at $\left.27.5 \mu \mathrm{m}\right)$. On an experimental point of view, a rather simple to achieve period is $27.5 \mu \mathrm{m}$ (corresponding to $p=4$ ).

The component corresponding to the zero order (birefringent case) arises naturally in a grating whose duty cycle is different from $50 \%$.

\subsection{Matching of the production rates of the pairs}

As wrotten previously, the amplitude of the two-photon state is proportional to $\tilde{\chi}_{i j k}^{(2)}(\mathbf{q})$. The condition of equal production of pairs in the same spatial mode by both interactions is

$$
\left|\tilde{\chi}_{e e e}^{(2)}(\mathbf{q})\right|^{2}=\left|\tilde{\chi}_{e o o}^{(2)}(\mathbf{0})\right|^{2}
$$

Introducing the analytical expression of the Fourier coefficients of a square-wave function, the condition is now

$$
\left|\chi_{\text {eee }}^{(2)}\right|^{2}\left[\frac{8}{n} \sin (n \alpha)\right]^{2}=\left|\chi_{\text {eoo }}^{(2)}\right|^{2}[4(2 \alpha-\pi)]^{2},
$$

where $\alpha$ is the duty cycle of the poling profile in radians ( $\alpha$ is defined as pointed out on Fig.1). Setting $n=4$ gives a duty cycle $\alpha \approx \pi / 6$. The theoretical parameters of a 1D PPLN enabling maximally polarization entangled pairs $(810 \mathrm{~nm}, 1550 \mathrm{~nm})$ from a $532-\mathrm{nm}$ pump beam at $100{ }^{\circ} \mathrm{C}$ are:

$$
\begin{aligned}
& \Lambda=27.5 \mu \mathrm{m} \\
& \alpha \approx 0.5 \mathrm{rad} .
\end{aligned}
$$




\section{BI-DIMENSIONAL PPLN FOR FREQUENCY ENTANGLEMENT}

Generalizing the possibility of having multiple phase-matching conditions in one-dimensional PPLN, we investigate here the possibility to use bi-dimensional PPLN as a source of frequency entangled states. Hexagonally 2-D PPLN structures have been used in the past few years as efficient crystals for multiple harmonic generation. ${ }^{12}$ However, to our knowledge no such structure has been experimentally used in an SPDC configuration. We report in the following the theoretical engineering of a 2D PPLN for generating frequency entangled pairs.

\subsection{Engineering frequency entangled pairs from a $2 \mathrm{D}$ nonlinear photonic crystal}

For the same reasons as described in the previous section, our aim is to create (810 nm, $1550 \mathrm{~nm})$ photon pairs from a 532-nm pump beam. The intended frequency entangled state is

$$
\frac{1}{\sqrt{1+|\zeta|^{2}}}\left(|810\rangle_{1}|1550\rangle_{2}+\zeta|1550\rangle_{1}|810\rangle_{2}\right) .
$$

This state requires matching the direction of emission of the 810-nm photon from the first pair with the 1550 -nm photon from the second one, and vice versa. The nonlinear processes considered here are type I where all waves are $e$-polarized, in order to use the highest nonlinear coefficient of lithium niobate.

The vectorial conditions are :

$$
\begin{aligned}
& \mathbf{k}_{532}=\mathbf{k}_{\mathbf{8 1 0}}^{\mathbf{1}}+\mathbf{k}_{1550}^{1}+\mathbf{k}^{1} \\
& \mathbf{k}_{\mathbf{5 3 2}}=\mathbf{k}_{\mathbf{8 1 0}}^{2}+\mathbf{k}_{1550}^{2}+\mathbf{k}^{2} \\
& \mathbf{k}_{\mathbf{8 1 0}}^{1} \| \mathbf{k}_{1550}^{2} \\
& \mathbf{k}_{\mathbf{8 1 0}}^{2} \| \mathbf{k}_{1550}^{1}
\end{aligned}
$$

where the superscripts 1 and 2 refer to the two photon pairs. The quantities $\mathbf{k}^{\mathbf{1}}$ and $\mathbf{k}^{\mathbf{2}}$ are two vectors of the reciprocal lattice of the $2 \mathrm{D}-\mathrm{PPLN}$. We choose the frame of a $2 \mathrm{D}$ PPLN with a poling pattern symmetric with respect to the direction of the pump beam (x-axis). In this way, the vectors $\mathbf{k}^{\mathbf{1}}$ and $\mathbf{k}^{\mathbf{2}}$ and the two nonlinear processes are symmetric, as depicted in Fig.2. This ensures that the production rates of the pairs by both interactions are the same in order to have $|\zeta|=1$ (maximally-entangled state). Using the Sellmeier equations, we compute the directions of output of the generated $(810 \mathrm{~nm}, 1550 \mathrm{~nm})$ pairs in a bidimensional PPLN. The general shape consists in two systems of bi-cones with a slight angle between them. The parameters of the poling can be chosen so that the 810-cone from the first interaction is made tangent with the 1550-one from the second interaction, and vice-versa. The theoretical output of the two processes is represented on Fig.2. This condition does not lead to a unique couple $\left(\mathbf{k}^{\mathbf{1}}, \mathbf{k}^{\mathbf{2}}\right)$, but we chose an easily achievable set of poling periods, that is to say a longitudinal period of the $2 \mathrm{D}$ poling equal to $7.2 \mu \mathrm{m}$ and a transverse one equal to $32 \mu \mathrm{m}$. As small poling patterns are more difficult to achieve, we chose as the longitudinal period $21.5 \mu \mathrm{m}$, whose third spatial harmonics is at $7.2 \mu \mathrm{m}$.

\section{CONCLUSION}

In this paper we propose the use of periodically poled lithium niobate as a source of polarization and frequency entangled pairs. Both of them use two generation processes (two different type I processes in a one-dimensional PPLN in the first case, the same type I process in a bi-dimensional PPLN for the second one). These crystals are promising materials for further quantum optics experiments, including Bell-state measurements, entanglement swapping and experimental realizations at the telecommunications wavelength.

\section{ACKNOWLEDGMENTS}

This work was supported by the National Science Foundation (NSF); by the Center for Subsurface Sensing and Imaging Systems (CenSSIS), an NSF Engineering Research Center; by the Defense Advanced Research Project Agency (DARPA); and by the David and Lucile Packard Foundation. 

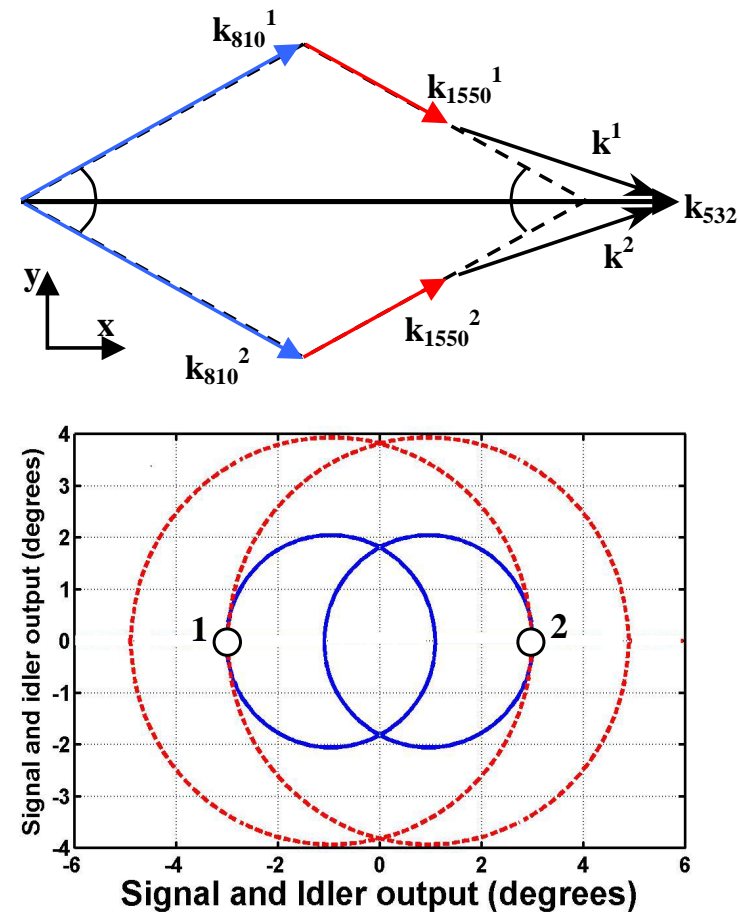

Figure 2. Top: Phase-matching diagram for a frequency-entangled bi-dimensional PPLN structure. Bottom: Computed output of a 2D PPLN structure at $150{ }^{\circ} \mathrm{C}$ with longitudinal and transverse poling periods equal to 21.5 and $32 \mu \mathrm{m}$ respectively. The small and large rings correspond to the 810-nm and 1550-nm photons, respectively . Frequency entangled pairs are collected along the directions labelled 1 and 2 .

\section{REFERENCES}

1. D. N. Klyshko, Photons and Nonlinear Optics, Gordon and Breach (New York, 1988).

2. P. G. Kwiat, K. Mattle, H. Weinfurter, A. Zeilinger, A. V. Sergienko, and Y. H. Shih, Phys. Rev. Lett. 75, 4337 (1995).

3. P. G. Kwiat, E. Waks, A. G. White, I. Appelbaum, and P. H. Eberhard, Phys. Rev. A 60, R773 (1999).

4. P. N. Butcher, The Elements of Nonlinear Optics, Cambridge University Press (Cambridge, 1990).

5. Y. R. Shen, The Principles of Nonlinear Optics, Wiley (New York, 1984).

6. J. A. Armstrong, N. Bloembergen, J. Ducuing, and P. S. Pershan, Phys. Rev. 127, 1918 (1962).

7. P. A. Franken and J. F. Ward, Rev. Mod. Phys. 35, 23 (1963).

8. L. E Myers, R. C. Eckart, M. M. Fejer, R. L. Byer, W. R. Rosenberg, and J. W. Pierce, J. Opt. Soc. Am. B 12, $2102(1995)$.

9. M. M. Fejer, G. A. Magel, D. H. Jundt, and R. L. Byer, IEEE J. Quantum Electron. 28, 2631 (1992).

10. G. D. Miller, R. G. Batchko, W. M. Tulloch, D. R. Weise, M. M. Fejer, and R. L. Byer, Opt. Lett. 22, 1834 (1997).

11. S. Tanzilli, H. de Riedmatten, W. Tittel, H. Zbinden, P. Baldi, M. De Micheli, D. B. Ostrowsky, and N. Gisin, Electron. Lett. 37 (2001).

12. N. G. R Broderick, G. W. Ross, H. L. Offerhaus, D. J. Richardson, and D. C. Hanna, Phys. Rev. Lett. 84, 4345 (2000). 